\title{
In-situ STEM Metallization of DNA Origami
}

$\underline{\text { Sardar Bilal Alam }}^{1}$, Pierre E. Palo ${ }^{1,2}$, Lee Bendickson ${ }^{1,2}$, Md Monirul Islam $^{1,3}$, Andrew C. Hillier ${ }^{1,3}$, Marit Nilsen-Hamilton ${ }^{1,2}$, and Tanya Prozorov ${ }^{1}$

${ }^{1}$ US DOE Ames Laboratory, Division of Materials Science and Engineering, Ames, USA.

${ }^{2}$ Iowa State University, Roy J. Carver Department of Biochemistry Biophysics and Molecular Biology, Ames, USA.

${ }^{3}$ Iowa State University, Department of Chemical and Biological Engineering, Ames, USA.

Soft matter nanoparticles can be synthesized in a wide range of complex geometries and are, hence, useful as "soft" templates for the synthesis of "hard" inorganic nanoparticles. This templating strategy offer room-temperature pathways to a variety of functional nanostructures with shapes and sizes not realizable via conventional inorganic chemical techniques. Among the soft templates, DNA origami [1] due to their low-cost, unprecedented highly-controlled assembly and site-specific binding ability, have gained much interest for bottom-up assembly. For example, metallized DNA origami can be used to make optical metamaterials [2]. However, the mechanism of DNA-templated nanoparticle nucleation remains elusive and fundamental understanding is lacking on how the DNA template dictates nanoparticle formation, attachment, growth, and ultimately, functional properties. Hence it is a challenge to construct geometrically precise metallized layers on DNA origami with high repeatability [3-5].

Here we use liquid phase in situ scanning transmission electron microscopy (STEM) to study and optimize the gold metallization of DNA origami triangles with the sides of 128 nanometers. To metallize the DNA origami triangles with Au, we start by introducing 1-2 nm Ag or Pd seeds on the origami structures. This seeding process is followed by either e-beam- or chemical agent- driven reduction of an Au precursor in the liquid phase in situ. We discuss the size, location of the seeds on the DNA origami, as well as the seeding density per triangle, and their effect on the morphology and quality of the formed Au metal coverage. Further, we demonstrate how the rate of Au precursor reduction and attachment to the surface determines the morphology of the triangles. Finally, we outline strategies for synthesis of metal nanostructure that maps the shape of the original DNA template with a continuous and uniform metal layer [6].

References:

[1] PWK Rothemund, Nature 440 (2006), p. 297.

[2] MM Hossen et al, Nanotechnology 29 (2018), p. 355603.

[3] J Richter et al, Advanced Materials 12 (2000), p. 507

[4] K Ijiro et al, E-Journal of Surface Science and Nanotechnology 3 (2005), p. 82.

[5] K Ijiro and H Mitomo, Polymer Journal 49 (2017), p. 815.

[6] This work was supported by the U.S. Department of Energy (DOE), Office of Science, Basic Energy Sciences, Division of Materials Sciences and Engineering. The research was performed at the Ames Laboratory, which is operated for the U.S. Department of Energy by Iowa State University under Contract No. DE-AC02-07CH11358. 

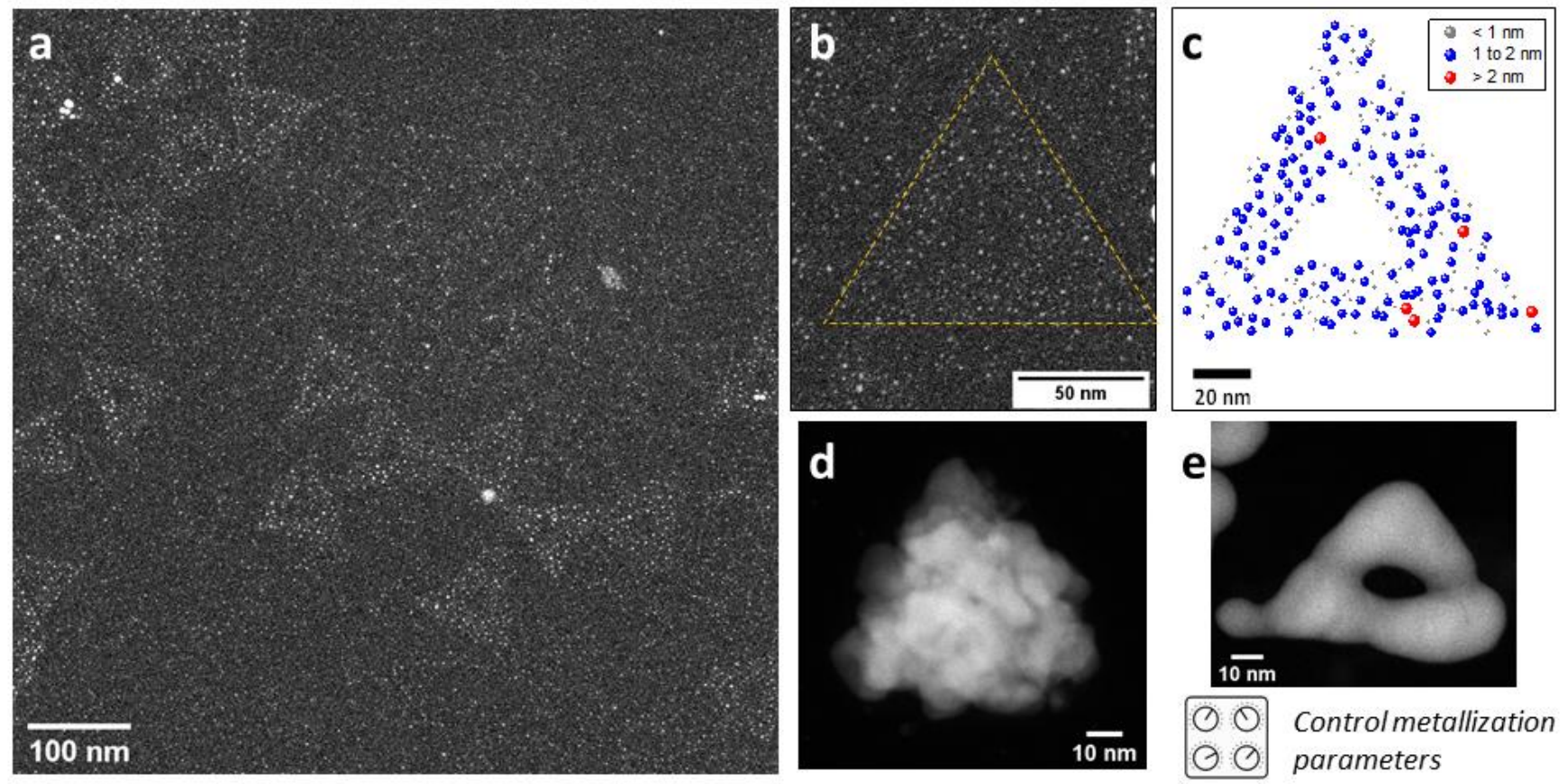

Figure 1. (a) STEM image of multiple DNA origami triangles with Ag seeds prior to Au metallization. (b) STEM image and corresponding (c) map of Ag seeds locations on a DNA-origami triangle. STEM image of Au metallized DNA origami triangles at (d) higher and (e) lower Au reduction rate and with different starting Ag seed population. 